\title{
A Systematic Review of Clinical Decision Support Systems for Clinical Oncology Practice
}

Pamala A. Pawloski, PharmDª, Gabriel A. Brooks, MD; Matthew E. Nielsen, MD'; and Barbara A. Olson-Bullis, MA

\begin{abstract}
Background: Electronic health records are central to cancer care delivery. Electronic clinical decision support (CDS) systems can potentially improve cancer care quality and safety. However, little is known regarding the use of CDS systems in clinical oncology and their impact on patient outcomes. Methods: A systematic review of peer-reviewed studies was performed to evaluate clinically relevant outcomes related to the use of CDS tools for the diagnosis, treatment, and supportive care of patients with cancer. Peer-reviewed studies published from 1995 through 2016 were included if they assessed clinical outcomes, patient-reported outcomes (PROs), costs, or care delivery process measures. Results: Electronic database searches yielded 2,439 potentially eligible papers, with 24 studies included after final review. Most studies used an uncontrolled, prepost intervention design. A total of 23 studies reported improvement in key study outcomes with use of oncology CDS systems, and 12 studies assessing the systems for computerized chemotherapy order entry demonstrated reductions in prescribing error rates, medicationrelated safety events, and workflow interruptions. The remaining studies examined oncology clinical pathways, guideline adherence, systems for collection and communication of PROs, and prescriber alerts. Conclusions: There is a paucity of data evaluating clinically relevant outcomes of CDS system implementation in oncology care. Currently available data suggest that these systems can have a positive impact on the quality of cancer care delivery. However, there is a critical need to rigorously evaluate CDS systems in oncology to better understand how they can be implemented to improve patient outcomes.
\end{abstract}

J Natl Compr Canc Netw 2019;17(4):331-338 doi: 10.6004/jnccn.2018.7104

${ }^{a}$ HealthPartners Institute, Minneapolis, Minnesota; ${ }^{b}$ The Health Care Systems Research Network (HCSRN) Cancer Research Network (CRN); 'DartmouthHitchcock Medical Center, Lebanon, New Hampshire; and University of North Carolina School of Medicine, Lineberger Comprehensive Cancer Center, Chapel Hill, North Carolina.

\section{Background}

Clinical decision support (CDS) systems include any electronic system designed to directly aid clinical decisionmaking by using individual patient characteristics to generate patient-specific assessments or recommendations. ${ }^{1,2}$ These systems require computable biomedical knowledge, person-specific data, and a reasoning or inferencing mechanism that combines knowledge and data to generate and present information to clinicians as care is being delivered. ${ }^{3}$ Examples of CDS tools include computerized alerts and reminders, clinical guidelines, condition-specific order sets, focused patient data reports, documentation templates, diagnostic support, and contextually relevant reference information. Thus, CDS has the potential to drive evidence-based standardization of cancer care, improving care delivery and patient outcomes. CDS tools have been incorporated across the patient care spectrum, encompassing prevention, diagnosis, and clinical monitoring. Common roles for CDS include computerized physician order entry (CPOE) and electronic health record (EHR) clinical reminder systems. ${ }^{2}$

CDS improves healthcare process measures; however, data demonstrating their effectiveness on clinical outcomes and costs are limited. ${ }^{1}$ Accordingly, real-world uptake of CDS systems has been modest at best. ${ }^{4}$ Benefits of CDS include improved efficiency and quality of healthcare delivery and access to medical data; enhanced communication; and potential cost savings. ${ }^{5-14}$ In 2007, the American Medical Informatics Association (AMIA) sounded a call to action regarding CDS implementation that included 3 pillars for fully realizing the promise of CDS: (1) best knowledge available when needed; (2) high adoption and effective use; and (3) continuous improvement of knowledge and CDS methods. ${ }^{4}$ Furthermore, the Agency for Healthcare Research and Quality stated that the question is not whether CDS systems should be designed and implemented, but rather how to make it easy to do the right thing. ${ }^{15}$ Nevertheless, effective implementation of a

See JNCCN.org for supplemental online content. 
CDS system is a major undertaking, considering the vast amount of clinical data and its variability, availability, and structure across facilities.

Clinical oncology is a dynamic, multidimensional healthcare specialty with complex decision-making and care coordination needs and multiple handoffs between primary and specialty care providers. ${ }^{16}$ In 2013 , the Institute of Medicine reported that the cancer care delivery system was in crisis due to a lack of patient-centric care, palliative care, and evidence-based decision-making. ${ }^{17}$ CDS systems have the potential to significantly improve cancer care delivery, but there are critical gaps in the availability and use of effective CDS tools. ${ }^{18}$ To better understand the current landscape of CDS systems in oncology practice, we conducted a systematic review of the literature describing real-world implementation of CDS tools for the diagnosis, treatment, and supportive care of patients with cancer. Our objective was to investigate the reported impact of CDS systems on clinically relevant patient outcomes.

\section{Methods}

\section{Study Rationale and Definition}

We critically appraised and synthesized the published medical literature to answer the objective question, "What evidence supports the use of CDS systems for diagnosis, treatment, and supportive care in clinical oncology?" A CDS system was defined as any electronic system in which characteristics of individual patients are used to generate patient-specific assessments or recommendations that are then presented to clinicians to help with clinical decision-making. ${ }^{1}$

\section{Inclusion and Exclusion Criteria}

Inclusion/exclusion criteria were determined a priori. We performed a systematic review of peer-reviewed, English-language studies published between January 1, 1995, and December 31, 2016, that evaluated the implementation of an electronic CDS system in the field of cancer care. Included studies assessed $\geq 1$ of the following: clinical outcomes (including patient-reported outcomes [PROs]), costs, and care delivery process measures (eg, medication prescribing error rates or compliance with clinical practice guidelines [CPGs]). Studies of decision aids intended primarily for patient use were excluded. Of the 120 studies identified before 1995, none met the predefined inclusion criteria and therefore they were not included in this analysis.

\section{Literature Search Strategy}

In a manner consistent with the Preferred Reporting Items for Systematic Reviews and Meta-Analyses (PRISMA) statement, we conducted a comprehensive review of the clinical and scientific literature. ${ }^{19}$ A trained health sciences librarian (B.A.O.B.) performed a comprehensive search of the literature to identify studies meeting the inclusion criteria. Databases searched were: PubMed, Embase, Academic Search Premier, Web of Science, and Inspec. Relevant narrative reviews, systematic reviews, and meta-analyses were also evaluated for background information, but were not included in this study.

In PubMed, the MeSH terms defined the concepts of cancer and CDS systems, medical order entry systems, or clinical pathways. For optimal retrieval, all terms were supplemented with relevant title and text words. Full PubMed search parameters are available in supplemental eAppendix 1 (available with this article at JNCCN.org). Search strategies for Embase, Academic Search Premier, Web of Science, and Inspec were adjusted for the syntax appropriate for each database. Published reports in the peer-reviewed literature were identified, and all abstracts were reviewed for eligibility. Fulltext articles were retrieved and reviewed when additional information was needed. Bibliographies from selected key articles, relevant review articles, and related meta-analyses were reviewed to identify additional publications.

\section{Screening and Full-Text Data Extraction}

The study team made every effort to identify all publications meeting the inclusion criteria. Two investigators reviewed each title and abstract for potential inclusion. Titles and abstracts were excluded based on mutual agreement; those not mutually determined to be included or excluded were adjudicated by the third investigator. Each publication that was not excluded after review of the title and abstract was then subjected to a second round of full-text review by 2 members of the study team in the same manner as before, again with adjudication of discordance by the third investigator.

Data from included studies were abstracted from full-text publications using a data abstraction form that included the study objective and design, intervention, number of subjects, study results and conclusions, location of study by country, number of study facilities (single site vs multisite), setting (inpatient vs outpatient), patient diagnosis, subject age range, CDS tool type (stand-alone, EHR-embedded) and name, study or system features, funding source, and conflict of interest declaration.

\section{Results}

Electronic database searches yielded 2,439 potentially eligible papers (Figure 1). After review and screening of titles and abstracts, 83 full-text articles were assessed for eligibility, and 24 studies were included in the final analysis. 


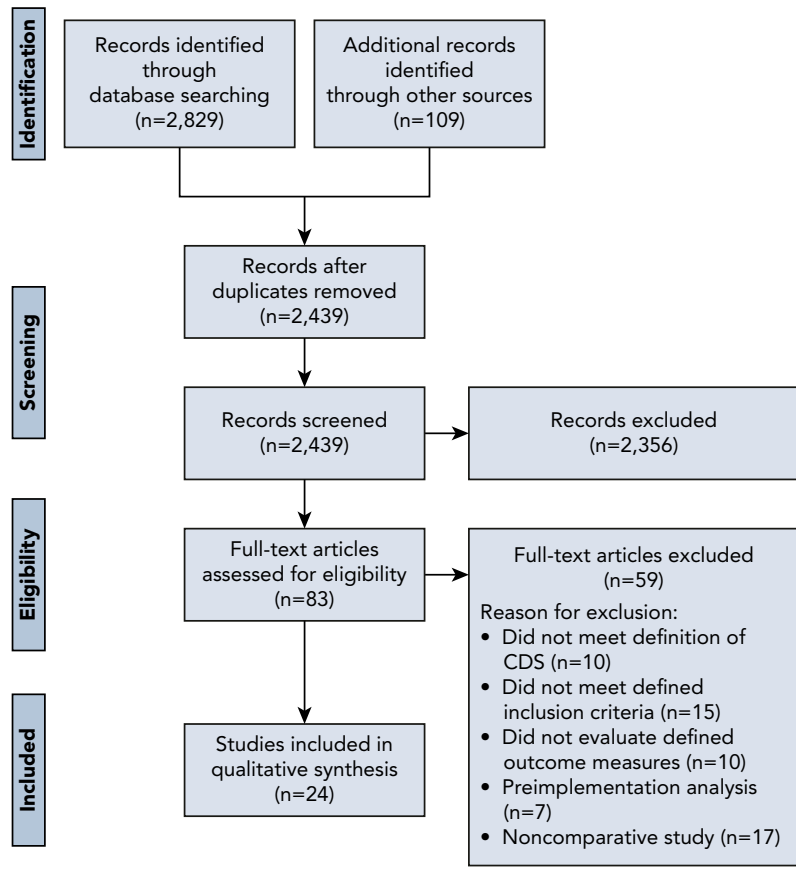

Figure 1. CONSORT diagram of studies showing evidence supporting the use of electronic clinical decision support (CDS) systems in cancer care.

Table 1 describes the characteristics of the included studies. Most were conducted in adults (96\%) in the United States (38\%) or Europe $(38 \%)$ and across any cancer diagnosis (71\%), and evaluated a variety of outcomes, including patients, clinicians, and clinician hours. The most common study outcomes were chemotherapy orders $(\mathrm{N}=76,619)$ or chemotherapy order sets $(\mathrm{N}=9,838)$. Most studies were conducted at a single facility (79\%), 87\% used quasi-experimental designs, and only $13 \%(n=3)$ were randomized controlled trials. Table 2 describes individual study sample sizes, study design, number of facilities, and diagnoses, and supplemental eTable 1 summarizes the objectives and outcomes of each included study.

Rate of prescription errors was the primary study outcome in 9 of the 12 studies evaluating CPOE. ${ }^{20-31}$ Although errors were defined differently among studies, prescription errors were reduced in all of them ${ }^{20,23-25,27-31}$ : 1 evaluated medication-related safety events, demonstrating fewer events with use of $\mathrm{CDS}^{22} ; 2$ evaluated pharmacy workflow, with 1 showing decreased workflow fragmentation and increased continuous task time with $\mathrm{CPOE}^{26}$; and another study showed increased order review time with CPOE. $^{21}$

CDS systems that could be classified as clinical pathways for care delivery processes were assessed in 6 of the 24 evaluated studies. ${ }^{32-37}$ The primary outcome for each of these studies was the association of the clinical pathway CDS system with receipt of guideline-concordant or pathway-recommended care. In general, the outcomes associated with use of clinical pathway systems were compared with usual care; however, one study compared use of an electronic clinical pathway with a paper pathway. ${ }^{34}$ Most studies were framed as reporting favorable outcomes, including reduced acute care use, ${ }^{32,35}$ increased guideline concordance and reduced symptoms, ${ }^{33}$ improved identification of eligible subjects for clinical trial participation, ${ }^{36}$ and improved hemoglobin levels among patients with anemia. ${ }^{37}$ The comparison between web- and paper-based pathway systems did not show a significant difference in pathway deviations between them. ${ }^{34}$

The remaining 6 studies assessed CDS systems in CPGs $(n=2),{ }^{38,39}$ PROs $(n=3),{ }^{40-42}$ and oncology-specific prescriber alerts $(n=1) .{ }^{43}$ One study evaluated CPG concordance with CDS and demonstrated a significant increase in guideline adherence $(P<.0001)$ accompanied by treatment plan modification in $31 \%$ of cases, and a $50 \%$ increase in clinical trial accrual. ${ }^{38}$ Furthermore, adherence to CPGs with CDS system advice was $>90 \%$ when the CDS tool included CPG recommendations. ${ }^{39}$ CDS tools for obtaining PROs and reporting the results to clinicians showed increased discussion of symptoms and quality-of-life issues $(P=.03)$ and symptom monitoring during routine clinical care. ${ }^{40-42}$ Patients reported a high ease of use and minimal time required; however, patient satisfaction was similar between intervention and control groups. One study evaluated prescriber alerts with CDS tools and demonstrated that hard stops for hepatitis B screening before chemotherapy treatment were associated with increased screening $(99.3 \%$ vs $40.2 \% ; P<.001)$ and chemoprophylaxis rates $(95.8 \%$ vs $39.2 \%$; $P<.001$ ) and a reduction in severe exacerbations of liver disease. ${ }^{43}$

\section{Discussion}

This systematic review assessed evidence supporting the use of CDS systems in cancer care delivery from studies evaluating cancer diagnosis, treatment, and supportive care. Importantly, most of our findings were consistent with those of previous non-oncology-focused studies showing that CDS systems improve care process measures. However, one study did not demonstrate favorable findings, and some of the findings were not of significant magnitude. ${ }^{28}$ Overall, these data demonstrate the potential value that CDS systems can bring to oncology clinical practices.

The largest category of studies included in our review comprises studies evaluating the use of CPOE systems in oncology care. These studies demonstrated a positive impact on prescriber errors, safety events, and workflow. ${ }^{20-31}$ CDS tools implementing clinical 


\begin{tabular}{|c|c|c|}
\hline Characteristic & $\mathbf{N}$ & $\%$ \\
\hline Full text articles included in analysis & 24 & 100 \\
\hline \multicolumn{3}{|l|}{ Study population } \\
\hline Adult & 23 & 96 \\
\hline Pediatric & 1 & 4 \\
\hline \multicolumn{3}{|l|}{ Cancer diagnosis } \\
\hline Any cancer diagnosis & 17 & 71 \\
\hline Multiple cancer diagnoses included & 2 & 8 \\
\hline Breast & 3 & 13 \\
\hline Prostate & 1 & 4 \\
\hline Renal cell & 1 & 4 \\
\hline \multicolumn{3}{|l|}{ Outcome samples studied } \\
\hline Patients & 5,730 & - \\
\hline Clinicians & 276 & - \\
\hline Clinician hours & 228 & - \\
\hline Decisions & 521 & - \\
\hline Chemotherapy orders & 76,619 & - \\
\hline Chemotherapy order sets & 9,838 & - \\
\hline Medication-related events & 212 & - \\
\hline Radiation therapy courses & 7,904 & - \\
\hline \multicolumn{3}{|l|}{ Study design } \\
\hline Randomized controlled trial & 3 & 13 \\
\hline Nonrandomized & 21 & 87 \\
\hline Pre-post & 10 & 48 \\
\hline Cross-sectional & 7 & 33 \\
\hline Other & 4 & 19 \\
\hline \multicolumn{3}{|l|}{ Number of facilities } \\
\hline Single & 19 & 79 \\
\hline Multisite & 5 & 21 \\
\hline \multicolumn{3}{|l|}{ Study setting } \\
\hline Inpatient & 7 & 29 \\
\hline Outpatient & 5 & 21 \\
\hline Not specified & 12 & 50 \\
\hline \multicolumn{3}{|l|}{ CDS system type } \\
\hline CPOE & 12 & 50 \\
\hline Clinical pathway & 6 & 25 \\
\hline CPG & 2 & 8 \\
\hline PROs & 3 & 13 \\
\hline Prescriber alert & 1 & 4 \\
\hline \multicolumn{3}{|l|}{ CDS tool type } \\
\hline Integrated into EHR & 13 & 54 \\
\hline Integrated into other system & 1 & 4 \\
\hline Internet-based & 2 & 8 \\
\hline Stand-alone & 8 & 33 \\
\hline
\end{tabular}

(continued)

\begin{tabular}{|c|c|c|}
\hline Characteristic & $\mathbf{N}$ & $\%$ \\
\hline \multicolumn{3}{|l|}{ Country } \\
\hline United States & 9 & 38 \\
\hline Europe & 9 & 38 \\
\hline Canada & 1 & 4 \\
\hline Korea & 1 & 4 \\
\hline Pakistan & 1 & 4 \\
\hline Taiwan & 3 & 13 \\
\hline \multicolumn{3}{|l|}{ Study funding source } \\
\hline Not specified & 12 & 50 \\
\hline $\mathrm{NIH}$ & 2 & 8 \\
\hline Other national program & 4 & 17 \\
\hline Foundation & 2 & 8 \\
\hline Industry & 1 & 4 \\
\hline Local/Facility & 3 & 13 \\
\hline \multicolumn{3}{|l|}{ Conflict of interest disclosure } \\
\hline Not reported & 10 & 42 \\
\hline None & 11 & 46 \\
\hline Affiliated with for-profit entity & 3 & 13 \\
\hline
\end{tabular}

Abbreviations: CDS, clinical decision support; $C P G$, clinical practice guideline; CPOE, computerized provider order entry; EHR, electronic health record; PROs, patient-reported outcomes.

pathways, ${ }^{32-37} \mathrm{CPGs}^{38,39} \mathrm{PROs}^{40-42}$ and prescriber alerts ${ }^{43}$ were also primarily associated with positive outcomes, including reduced hospital stays, ${ }^{35}$ increased guideline use and concordance, ${ }^{33,38,39}$ enhanced identification of trialeligible patients, ${ }^{36}$ and improvements in symptom management. ${ }^{41}$ Overall, the studies suggest that clinical oncology-focused CDS systems appear to be well accepted and are associated with potentially meaningful improvements in patient care.

Two systematic reviews that assessed CPOE for inpatients in medicine or intensive care units ${ }^{12}$ and at the point of care in any clinical setting ${ }^{13}$ demonstrated a clear benefit to implementing CDS. However, one study in our analysis showed that there was an increase in pharmacist order review time without any impact on intervention rates. ${ }^{21}$ This finding underlines the potential for CDS systems to have a negative impact on care delivery, and demonstrates the importance of a thorough evaluation of these tools as a part of system implementation.

CDS systems incorporated into clinical pathways have been associated with increased guideline adherence, ${ }^{32-37}$ demonstrating the benefit they can provide to clinicians, and consistent with findings of previous systematic reviews also showing a positive impact on guideline adherence. ${ }^{10,44}$ 
Table 2. Study Characteristics

\begin{tabular}{|c|c|c|c|c|}
\hline Study & Sample Size & Study Design ${ }^{51}$ & $\begin{array}{l}\text { Number of } \\
\text { Facilities }\end{array}$ & $\begin{array}{l}\text { Cancer } \\
\text { Diagnosis }\end{array}$ \\
\hline Aziz et al, $2015^{20}$ & 9,279 chemotherapy orders & Pre-post, no control group & Single & Any \\
\hline Basch et al, $2016^{42}$ & 766 patients & Randomized controlled trial & Single & Multiple \\
\hline Beer et al, $2002^{21}$ & 836 chemotherapy orders & Nonrandomized & Multiple & Any \\
\hline Beriwal et al, $2012^{32}$ & 7,904 radiation therapy courses & Pre-post, no control group & Multiple & Multiple \\
\hline Berry et al, $2011^{40}$ & 765 patients, 262 clinicians & Randomized controlled trial & Multiple & Any \\
\hline Bertsche et al, $2009^{33}$ & 100 patients & Pre-post, uncontrolled & Single & Any \\
\hline Bouaud et al, $2001^{38}$ & 127 decisions & Cross-sectional with internal control & Single & Breast \\
\hline Bouaud et al, $2015^{39}$ & 394 decisions & Cross-sectional with internal control & Multiple & Breast \\
\hline Chang et al, $2002^{34}$ & 124 patients & Pre-post, no control group & Single & Renal cell \\
\hline Chen \& Lehmann, $2011^{22}$ & 212 medication-related events & Pre-post, no control group & Single & Any pediatric \\
\hline Cho et al, $2013^{23}$ & 54,561 chemotherapy orders & Cross-sectional with nonequivalent control & Single & Any \\
\hline Collins \& Elsaid, $2011^{24}$ & 538 chemotherapy orders & Pre-post, no control group & Single & Any \\
\hline Elsaid et al, $2013^{25}$ & 1,192 chemotherapy orders & Interrupted time series, no control group & Single & Any \\
\hline Hanauer et al, $2013^{26}$ & 228 clinician hours & Pre-post, no control group & Single & Any \\
\hline Hsu et al, $2008^{35}$ & 44 patients & Pre-post, no control group & Single & Prostate \\
\hline Hsu et al, $2015^{43}$ & 2,512 patients & $\begin{array}{l}\text { Three-stage study (pre, post } 1, \text { post } 2) \text {, } \\
\text { no control group }\end{array}$ & Single & Any \\
\hline Huertas Fernández et al, 2006²7 & 60 chemotherapy orders & Cross-sectional with nonequivalent control & Single & Any \\
\hline Mattsson et al, $2015^{28}$ & 5,767 chemotherapy orders & Cross-sectional with nonequivalent control & Multiple & Any \\
\hline Meisenberg et al, $2014^{29}$ & 9,838 chemotherapy order sets & Quality improvement, no control group & Single & Any \\
\hline Patkar et al, $2012^{36}$ & 1,295 patient cases & Cross-sectional with internal control & Single & Breast \\
\hline Ruland et al, $2003^{41}$ & 14 clinicians, 56 patients & Randomized controlled trial & Single & Any \\
\hline Small et al, $2008^{30}$ & 1,941 chemotherapy orders & Cross-sectional with nonequivalent control & Single & Any \\
\hline Van Erps et al, $2010^{37}$ & 68 patients & Pre-post, no control group & Single & Any \\
\hline Voeffray et al, $2006^{31}$ & 2,445 chemotherapy orders & Pre-post, no control group & Single & Any \\
\hline
\end{tabular}

Three studies included in our analysis evaluated the use of CDS systems for PROs, and all demonstrated benefit for $\geq 1$ outcome. ${ }^{40-42}$ These findings differ somewhat from those of a systematic review of 15 studies that assessed the effect of CDS systems on PROs, which showed a positive effect on symptoms in 3 studies (20\%). ${ }^{45}$ A CDS system used with prescriber alerts demonstrated a positive impact, which is consistent with findings of a previous study. ${ }^{43,46}$

The findings are also consistent with those of a meta-analysis assessing the impact of health information technology (HIT) on cancer care from 2000 to June $2014 .{ }^{47}$ CDS systems were the most common (66\%) HIT intervention identified and were implemented across several cancer types, including breast, colorectal, and prostate, for detection, diagnosis, and treatment but not for survivorship or end-of-life care. The primary findings show that the beneficial impact differed across the cancer continuum; HIT for diagnosis and treatment was less likely to be associated with benefit compared with prevention. Likewise, HIT targeting behavioral change is less likely to be beneficial compared with HIT targeting improved decision-making. The complexity of diagnosis and treatment, the volume of information needed, and the factors associated with behavior change were given as potential reasons for these findings. Within the CDS systems, key factors that appear to contribute to improved outcomes include the use of real-time provider alerts and point-of-care action on prescription orders and provision of information to clinicians that CDS systems can provide. ${ }^{20,27,32,36,37,40-42}$ There appear to be resulting factors that create new challenges, such as the need to access separate systems and otherwise increase work time of prescribers or other downstream clinicians, that may reduce outcomes. ${ }^{21,26,34,35}$

Our systematic review is reported $>10$ years after a call to action by the AMIA regarding the use of CDS. ${ }^{4}$ The call included directives for achieving desirable levels of patient safety, care quality, patient-centeredness, and cost-effectiveness. Health systems were called on to 
optimize CDS to improve the quality of healthcare services and health in the United States. However, not all studies evaluating CDS systems have shown clinical practice improvements. ${ }^{48}$ Thus, it is imperative that systems and tools, both commercially and locally developed, be assessed for their effectiveness and impact on patient outcomes. Furthermore, we believe it is essential that these assessments also involve outcomes, including those associated with clinical care and costs of system implementation, when possible.

Kowamoto et $\mathrm{al}^{8}$ identified features of CDS systems critical for improving clinical practice in any clinical setting, including (1) automatic provision of decision support as part of clinician workflow; (2) provision of recommendations compared with assessments; (3) provision of decision support at the time and location of decision-making; and (4) computer-based decision support. CDS systems with all 4 features were associated with significant improvements in clinical practice.

A major gap in CDS system use exists across the spectrum of clinical oncology care, and further development of CDS tools is warranted. Findings from the limited studies included in this review are largely positive and consistent with most studies conducted in the general healthcare population. However, many of the included studies used suboptimal study designs, and only 3 were randomized controlled trials. Robust quasi-experimental study designs, such as interrupted time series designs with a comparator group, were rare. Currently, $<15$ studies assessing the impact of CDS systems are underway or accruing, according to ClinicalTrials.gov. ${ }^{49} \mathrm{~A}$ recent study of CDS in patients with lung cancer showed a reduction in inappropriate granulocyte colony-stimulating factor use without an increase in febrile neutropenia rates, illustrating the positive impact these powerful tools can have on clinical oncology care. ${ }^{50}$ Further analyses of the use of these tools with appropriate study designs and analytic methods are necessary to build the case for wider implementation of CDS systems.

\section{Directions for Future Research}

A number of gaps in the current literature were identified during this analysis, including a lack of information related to the specific barriers, facilitators, and implementation strategies associated with CDS system implementation. The studies did not specify the potential challenges facilities may encounter when implementing similar systems or describe identified best practices to support successful implementation or outcomes. In addition, the impact of CDS systems on patient mortality, healthcare costs, or costs associated with implementation and management was also not assessed. Furthermore, outcome assessments for each study did not include overall impact on patient mortality or the impact of system implementation on healthcare costs to the institution, payer, or patient. The costs of system maintenance, upgrades, or enhancements were also not described. These are all crucial elements that must be assessed and understood for optimal implementation to occur.

\section{Study Strengths and Limitations}

Strengths of this systematic review include the study team, comprising a research librarian, 2 practicing oncologists, and an oncology clinical pharmacist. The review was conducted according to the PRISMA statement to ensure appropriate methods were used. The potential exists for bias, including searching, exclusion criteria, assembling, and publication, although all efforts were made to minimize this where possible. The total number of CDS systems that have been studied, had no outcome improvement, and were subject to publication bias is unknown.

\section{Conclusions}

To date, few studies have evaluated CDS systems in oncology practice. Comparative studies that report outcomes of care include those of CDS systems with CPOE, clinical pathways, CPGs, PROs, and provider alerts. Published studies of CDS systems are largely associated with positive outcomes, and their findings consistent with those of studies conducted in the noncancer population. Key features that appear to support positive outcomes include real-time information and point-of-care action. CPOE showed a small impact on provider behavior, with general alerts and increased pharmacist order review times. Potential drawbacks may be the need to access separate systems and increased workflow for prescribers or other clinicians. None of the studies assessed the impact of CDS systems on patient survival. There is a critical need for CDS systems development and well-designed studies to demonstrate improvement in patient outcomes, including impact on survival and efficiency of cancer care delivery.

\section{Acknowledgments}

The authors wish to acknowledge the guidance of Terry Field and Diana Buist, members of the Health Care Systems (HCS) Cancer Research Network (CRN) and CRN Scholar Program Mentors, who contributed to the development of this project. The overall goal of the CRN is to conduct collaborative research to determine the effectiveness of preventive, curative, and supportive interventions for major cancers that span the natural history of those cancers among diverse populations and health systems. HealthPartners Institute is an Affiliate Member of the HCS Research Network. The authors would also 
like to acknowledge Mandy Fraser, Amanda Lacy, and Lauryn Davin, who provided technical assistance during the literature review process and with manuscript preparation and submission.

Submitted May 4, 2018; accepted for publication November 5, 2018.

Author contributions: Study concept and design: Pawloski, Brooks, Nielsen. Provision of study materials: Olson-Bullis. Acquisition and assembly of data:
Pawloski, Olson-Bullis. Data analysis and interpretation: Pawloski, Brooks, Nielsen. Manuscript preparation and final approval: All authors.

Disclosures: The authors have not received any financial consideration from any person or organization to support the preparation, analysis, results, or discussion of this article.

Funding: This work was supported by a grant from the NCI (U24 CA171524; $\mathrm{Pl}$ : L. Kushi), with no additional funding provided specifically for this work.

Correspondence: Pamala A. Pawloski, PharmD, HealthPartners Institute, 8170 33rd Avenue S, MS23301A, Bloomington, MN 55425.

Email: pamala.a.pawloski@healthpartners.com

\section{References}

1. Lobach D, Sanders GD, Bright TJ, et al. Enabling health care decisionmaking through clinical decision support and knowledge management. Evid Rep Technol Assess (Full Rep) 2012;203:1-784.

2. Castaneda C, Nalley K, Mannion C, et al. Clinical decision support systems for improving diagnostic accuracy and achieving precision medicine. J Clin Bioinforma 2015;5:4.

3. Clinical Decision Support. HealthlT.gov website. Available at: https:// www.healthit.gov/policy-researchers-implementers/clinical-decisionsupport-cds. Accessed March 13, 2018

4. Osheroff JA, Teich JM, Middleton B, et al. A roadmap for national action on clinical decision support. J Am Med Inform Assoc 2007;14:141-145.

5. Ash JS, McCormack JL, Sittig DF, et al. Standard practices for computerized clinical decision support in community hospitals: a national survey. J Am Med Inform Assoc 2012;19:980-987.

6. de Lusignan $\mathrm{S}, \mathrm{Chan} \mathrm{T}$. The development of primary care information technology in the United Kingdom. J Ambul Care Manage 2008;31: 201-210.

7. Roshanov PS, Fernandes N, Wilczynski JM, et al. Features of effective computerised clinical decision support systems: meta-regression of 162 randomised trials. BMJ 2013;346:f657.

8. Kawamoto K, Houlihan CA, Balas EA, et al. Improving clinical practice using clinical decision support systems: a systematic review of trials to identify features critical to success. BMJ 2005;330:765.

9. Garg AX, Adhikari NK, McDonald H, et al. Effects of computerized clinical decision support systems on practitioner performance and patient outcomes: a systematic review. JAMA 2005;293:1223-1238.

10. Chaudhry B, Wang J, Wu S, et al. Systematic review: impact of health information technology on quality, efficiency, and costs of medical care. Ann Intern Med 2006;144:742-752.

11. Bonnabry P, Despont-Gros C, Grauser D, et al. A risk analysis method to evaluate the impact of a computerized provider order entry system on patient safety. J Am Med Inform Assoc 2008;15:453-460.

12. Kaushal R, Shojania KG, Bates DW. Effects of computerized physician order entry and clinical decision support systems on medication safety: a systematic review. Arch Intern Med 2003;163:1409-1416.

13. Bright TJ, Wong A, Dhurjati R, et al. Effect of clinical decision-support systems: a systematic review. Ann Intern Med 2012;157:29-43.

14. eHealth for a Healthier Europe! Opportunities for a better use of healthcare resources. Available at: https://joinup.ec.europa.eu/sites/ default/files/document/2014-12/eHealth\%2Ofor\%20a\%2OHealthier\% 20Europe\%20-\%20Opportunities\%20for\%20a\%20better\%20use\% 20of\%20healthcare\%20resources.pdf. Accessed March 13, 2018.

15. Berner ES. Clinical Decision Support Systems: State of the Art. AHRQ Publication No. 09-0069-EF. Rockville, MD: Agency for Healthcare Research and Quality; 2009.

16. Clauser SB, Wagner EH, Aiello Bowles EJ, et al. Improving modern cancer care through information technology. Am J Prev Med 2011;40(5 Suppl 2): S198-207.

17. Levit L, Balogh E, Nass S, Ganz PA, eds. Delivering High-Quality Cancer Care: Charting a New Course for a System in Crisis. Washington, DC: The National Academies Press; 2013.

18. Seidman AD. Computer-assisted decision support in medical oncology: we need it now. The ASCO Post, April 10, 2016.

19. Moher D, Liberati A, Tetzlaff J, et al. Preferred reporting items for systematic reviews and meta-analyses: the PRISMA statement. PLoS Med 2009;6:e1000097

20. Aziz MT, Ur-Rehman T, Qureshi S, et al. Reduction in chemotherapy order errors with computerised physician order entry and clinical decision support systems. Health Inf Manag 2015;44:13-22.
21. Beer J, Dobish R, Chambers C. Physician order entry: a mixed blessing to pharmacy? J Oncol Pharm Pract 2002;8:119-126.

22. Chen AR, Lehmann CU. Computerized provider order entry in pediatric oncology: design, implementation, and outcomes. J Oncol Pract 2011;7: 218-222.

23. Cho E, Kim HJ, Kim GM, et al. Assessment of efficiency and safety of the comprehensive Chemotherapy Assistance Program for ordering oncology medications. Int J Med Inform 2013;82:504-513.

24. Collins CM, Elsaid KA. Using an enhanced oral chemotherapy computerized provider order entry system to reduce prescribing errors and improve safety. Int J Qual Health Care 2011;23:36-43.

25. Elsaid K, Truong T, Monckeberg M, et al. Impact of electronic chemotherapy order forms on prescribing errors at an urban medical center: results from an interrupted time-series analysis. Int J Qual Health Care 2013;25:656-663.

26. Hanauer DA, Zheng K, Commiskey EL, et al. Computerized prescriber order entry implementation in a physician assistant-managed hematology and oncology inpatient service: effects on workflow and task switching. J Oncol Pract 2013;9:e103-114

27. Huertas Fernández MJ, Baena-Cañada JM, Martínez Bautista MJ, et al. Impact of computerised chemotherapy prescriptions on the prevention of medication errors. Clin Transl Oncol 2006;8:821-825.

28. Mattsson TO, Holm B, Michelsen $\mathrm{H}$, et al. Non-intercepted dose errors in prescribing anti-neoplastic treatment: a prospective, comparative cohor study. Ann Oncol 2015;26:981-986.

29. Meisenberg BR, Wright RR, Brady-Copertino CJ. Reduction in chemotherapy order errors with computerized physician order entry. J Oncol Pract 2014;10:e5-9.

30. Small MD, Barrett A, Price GM. The impact of computerized prescribing on error rate in a department of Oncology/Hematology. J Oncol Pharm Pract 2008;14:181-187.

31. Voeffray M, Pannatier A, Stupp R, et al. Effect of computerisation on the quality and safety of chemotherapy prescription. Qual Saf Health Care 2006; 15:418-421.

32. Beriwal S, Rajagopalan MS, Flickinger JC, et al. How effective are clinical pathways with and without online peer-review? An analysis of bone metastases pathway in a large, integrated National Cancer InstituteDesignated Comprehensive Cancer Center Network. Int J Radiat Oncol Biol Phys 2012;83:1246-1251.

33. Bertsche T, Askoxylakis V, Habl G, et al. Multidisciplinary pain management based on a computerized clinical decision support system in cancer pain patients. Pain 2009;147:20-28.

34. Chang PL, Li YC, Lee SH. The differences in health outcomes between Web-based and paper-based implementation of a clinical pathway for radical nephrectomy. BJU Int 2002;90:522-528.

35. Hsu YC, Tsui KH, Chen CL, et al. Web-based clinical pathway for reducing practice variations in radical prostatectomy. Chang Gung Med J 2008;31: 567-575.

36. Patkar V, Acosta D, Davidson $T$, et al. Using computerized decision support to improve compliance of cancer multidisciplinary meetings with evidence-based guidance. BMJ Open 2012;2:2.

37. Van Erps J, Aapro M, MacDonald K, et al. Promoting evidence-based management of anemia in cancer patients: concurrent and discriminant validity of RESPOND, a web-based clinical guidance system based on the EORTC guidelines for supportive care in cancer. Support Care Cancer 2010;18:847-858.

38. Bouaud J, Séroussi B, Antoine EC, et al. A before-after study using OncoDoc, a guideline-based decision support-system on breast cancer 
management: impact upon physician prescribing behaviour. Stud Health Technol Inform 2001;84:420-424.

39. Bouaud J, Spano JP, Lefranc JP, et al. Physicians' attitudes towards the advice of a guideline-based decision support system: a case study with OncoDoc2 in the management of breast cancer patients. Stud Health Technol Inform 2015;216:264-269.

40. Berry DL, Blumenstein BA, Halpenny B, et al. Enhancing patient-provider communication with the electronic self-report assessment for cancer: a randomized trial. J Clin Oncol 2011;29:1029-1035.

41. Ruland CM, White T, Stevens M, et al. Effects of a computerized system to support shared decision making in symptom management of cancer patients: preliminary results. J Am Med Inform Assoc 2003;10:573-579.

42. Basch E, Deal AM, Kris MG, et al. Symptom monitoring with patientreported outcomes during routine cancer treatment: a randomized controlled trial. J Clin Oncol 2016;34:557-565.

43. Hsu PI, Lai KH, Cheng JS, et al. Prevention of acute exacerbation of chronic hepatitis $B$ infection in cancer patients receiving chemotherapy in a hepatitis B virus endemic area. Hepatology 2015;62:387-396.

44. Damiani G, Pinnarelli L, Colosimo SC, et al. The effectiveness of computerized clinical guidelines in the process of care: a systematic review. BMC Health Serv Res 2010;10:2.
45. Blum D, Raj SX, Oberholzer R, et al. Computer-based clinical decision support systems and patient-reported outcomes: a systematic review. Patient 2015;8:397-409.

46. Scott GP, Shah P, Wyatt JC, et al. Making electronic prescribing alerts more effective: scenario-based experimental study in junior doctors. J Am Med Inform Assoc 2011;18:789-798.

47. Tarver WL, Menachemi N. The impact of health information technology on cancer care across the continuum: a systematic review and metaanalysis. J Am Med Inform Assoc 2016;23:420-427.

48. Hunt DL, Haynes RB, Hanna SE, et al. Effects of computer-based clinical decision support systems on physician performance and patient outcomes: a systematic review. JAMA 1998;280:1339-1346.

49. U.S. National Library of Medicine. ClinicalTrials.gov. Available at: https:// clinicaltrials.gov/ct2/home. Accessed September 13, 2018.

50. Adeboyeje G, Agiro A, Malin J, et al. Reducing overuse of colonystimulating factors in patients with lung cancer receiving chemotherapy: evidence from a decision support-enabled program. J Oncol Pract 2017 13:e337-345.

51. Harris AD, McGregor JC, Perencevich EN, et al. The use and interpretation of quasi-experimental studies in medical informatics. J Am Med Inform Assoc 2006;13:16-23.

\section{Soft Tissue Sarcoma FREE Patient Webinar}

For people with soft tissue sarcoma, their caregivers, and their families

Tuesday, June 11, 2019 Wednesday, June 12, 2019

10:00 - 11:15 AM ET 4:30 - 5:45 PM ET

Join experts as they discuss treatment of soft tissue sarcoma.

This webinar is designed to help participants:

- Demonstrate an understanding of what soft tissue sarcoma is, and how it is diagnosed and staged

- Compare, discuss, and select treatment options with their doctor

- Discover the most recent advancements in soft tissue sarcoma care

- Identify and utilize supportive care and patient and caregiver resources

Each webinar will also include a Question \& Answer session. You may submit questions to the presenters during the webinar or in advance.

*Please note: This is a non-accredited activity.

\section{Visit NCCN.org/patientwebinars to register.}

Supporters

This activity is supported through the NCCN Foundation, by a contribution from our industry supporter Lilly and Company. NCCN independently develops and distributes the NCCN Guidelines for Patients. Our industry supporters do not participate in the development of the NCCN Guidelines for Patients and are not responsible for the content and recommendations contained therein.

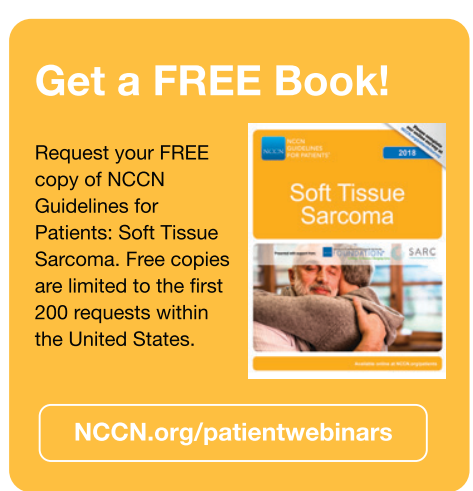

Endorsers

The NCCN Guidelines for Patients: Soft Tissue Sarcoma are endorsed and sponsored in part by: SARC (Sarcoma Alliance for Research through Collaboration) 